\title{
RECAI
}

RECAI Revista de Estudios en Contaduría,

Administración e Informática

ISSN: 2007-5278

danielarturoc@yahoo.com.mx

Universidad Autónoma del Estado de México

México

\section{Características del dueño-administrador en la innovación y desempeño de pequeñas y medianas empresas metalmecánicas de la Industria Automotriz del Valle de Toluca}

\author{
García Marín, Osvaldo; Nava Rogel, Rosa María \\ Características del dueño-administrador en la innovación y desempeño de pequeñas y medianas empresas \\ metalmecánicas de la Industria Automotriz del Valle de Toluca \\ RECAI Revista de Estudios en Contaduría, Administración e Informática, vol. 8, núm. 23, 2019 \\ Universidad Autónoma del Estado de México, México
}

Copyright 2019 Universidad Autónoma del Estado de México

Esta obra está bajo una Licencia Creative Commons Atribución-NoComercial-SinDerivar 4.0 Internacional. 
Artículos

\title{
Características del dueño-administrador en la innovación y desempeño de pequeñas y medianas empresas metalmecánicas de la Industria Automotriz del Valle de Toluca
}

\author{
Osvaldo García Marín valdovallo@live.com.mx \\ Universidad Autónoma del Estado de México, México \\ Rosa María Nava Rogel \\ Universidad Autónoma del Estado de México, México
}

RECAI Revista de Estudios en Contaduría, Administración e Informática, vol. 8, núm. 23, 2019

Universidad Autónoma del Estado de México, México

Recepción: 27 Julio 2019

Aprobación: 28 Agosto 2019

Copyright 2019 Universidad Autónoma del Estado de México

CC BY-NC-ND
Resumen: En este estudio se analiza la relación que existe entre las características del dueño-administrador y la innovación y el desempeño de las pequeñas y medianas empresas (PYMES) metalmecánicas del Valle de Toluca. Utilizando la tipología de orientación estratégica desarrollada por Miles y Snow (1978), se determina que en la muestra de pymes metalmecánicas participantes en esta investigación predominan los perfiles defensores y analistas. En ambos tipos se encontraron niveles similares de innovación y desempeño. Por otra parte, el rol que juega la persona que está dirigiendo la organización es la característica que presenta diferencias significativas. Esto es, los dueños tienen un mayor nivel de innovación y desempeño que los administradores. Se presentan algunas propuestas para las PYMES metalmecánicas de la industria automotriz del Valle de Toluca.

Palabras clave: Dueño, administrador, orientación estratégica, innovación, desempeño, PYMES.

Abstract: In this study we analyze the relationship between the characteristics of the owner-manager and the innovation and performance of metal-mechanic small and medium enterprises (SMEs) in Toluca Valley. Using the typology of strategic orientation developed by Miles and Snow (1978), we found that defender and analyst profiles predominate in the sample of metal-mechanic firms participating in this study. On the other hand, the role of the person who manages the organization is the characteristic that shows significant differences. That is, owners have a higher level of innovation and performance than managers. We posit some proposals for metalmechanic SMEs operating in the automotive industry in Toluca Valley.

Keywords: Owner, manager, strategic orientation, innovation, performance, SMEs.

\section{Introducción}

La internacionalización de la economía y los cambios frecuentes e inciertos a los que se enfrentan todos los días las organizaciones, han forzado a que éstas mejoren su desempeño. En particular, las pequeñas y medianas empresas (PYMES) sufren un mayor impacto, debido principalmente a sus economías de escala (van Stel, Lokshin y de Vries, 2019).

Es importante estudiar el contexto de las PYMES, pues a pesar de que tienen serias dificultades para sobrevivir y desarrollarse en un mercado 
cada vez más competitivo, su contribución en la generación de empleos, la obtención de ingresos y su papel como generadoras de riqueza, es evidente en todo el mundo (Estrada et al., 2009).

En México, las PYMES representan un motor que impulsa el desarrollo económico del país, ya que la gran mayoría de empresas existentes, pertenecen a este segmento (Navarrete, 2011). Las PYMES generan 72\% del empleo y 52\% del Producto Interno Bruto (PIB) (INEGI, 2015). Sin embargo, el $80 \%$ de ellas fracasa antes de los cinco años y $90 \%$ antes de cumplir una década (Vargas, 2012).

Las PYMES que se desarrollan dentro de la industria metalmecánica automotriz, se enfrentan a un entorno de mayor complejidad, por los cambios que se están promoviendo en la política exterior (Estañol et al., 2016) y por la fuerza de los fabricantes de automóviles asiáticos (Álvarez Medina, 2016). A pesar de ello, la industria automotriz en México continua siendo una de las principales fuentes de inversión extranjera directa, aportando el 3.5\% del PIB nacional y $16.9 \%$ del PIB manufacturero (INEGI y AMIA, 2016). Por ello, se requiere realizar estudios profundos sobres los fenómenos administrativos que afectan a las PYMES y desarrollar estrategias que les permita seguir permaneciendo en el mercado, considerando el perfil de sus dueños y/o administradores.

Día con día surgen estudios que explican las enormes diferencias que existen entre las pequeñas y las grandes empresas, haciendo evidente que las estrategias en las PYMES se centran en el dueño-administrador, dificultando la elaboración de planes para los siguientes años, lo que provoca cierta incertidumbre sobre el destino de la organización, por el poco o nulo personal calificado con el que cuentan, la estructura plana que impide una comunicación más fluida, recursos limitados, estrategias generales o improvisadas y la atención centrada en la operación diaria, entre otras (Baptista Nunes, et al., 2006).

A pesar de la vasta investigación sobre los factores particulares de los dueños-administradores asociados a la innovación y desempeño de las PYMES, existen pocos avances teóricos y generalizables. Los más destacados han propuesto el estudio de las características y la orientación estratégica del dueño y/o administrador como elementos clave para explicar el desempeño de las PYMES (Jaouen y Lasch, 2013), esperando mayor evidencia empírica que fortalezca esta postura.

Este artículo pretende contribuir a explicar la relación entre las características de los dueños administradores y el desempeño e innovación en PYMES, considerando los siguientes objetivos específicos:

1. Determinar el arquetipo estratégico que existe en el dueñoadministrador de la PYME del sector metalmecánico automotriz en el Valle de Toluca y sus diferencias significativas en cuanto a innovación y desempeño

2. Determinar las características del dueño o administrador de la PYME metalmecánica automotriz del Valle de Toluca que tienen mayor relación con la innovación y el desempeño. 
Para lograr los objetivos planteados se abordan cuatro secciones. En primer término se analiza la visión basada en recursos, para identificar los principales factores que, de acuerdo a los estudiosos en el tema, pueden ser una fuente de ventaja competitiva sostenible para las PYMES; utilizando la tipología de Miles y Snow (1978), se analizan los comportamientos de los diferentes arquetipos estratégicos (Prospector, Defensor, Analista y Reactor). En seguida, se aborda el contexto que viven las empresas que son parte de la cadena de proveeduría del sector metalmecánico automotriz en México, específicamente en las PYMES del Valle de Toluca. En tercer lugar, se describe el modelo de investigación. Finalmente y en cuarto lugar, se presentan los hallazgos, así como las conclusiones y propuestas.

Cabe resaltar que a pesar de que se abarcó a una gran parte de la población de las PYMES metalmecánicas automotrices instaladas en el Valle de Toluca, la tasa de respuesta fue del $46 \%$, pues los dueñosadministradores de las PYMES, expresaron su temor pensando que al contestar, pudiera haber repercusiones por las autoridades hacendarias o bien por la delincuencia organizada. Por ello, la muestra no fue lo suficientemente grande para realizar inferencias estadísticas que ayuden a generalizar los hallazgos encontrados.

\section{Teoría de recursos intangibles, capacidades y ventajas competitivas}

La teoría de recursos y capacidades (Barney 1991; Prahalad y Hamel 1990; Wernerfelt 1984), explica la importancia de los recursos y las capacidades que posee la empresa para mejorar sus oportunidades y hacer frente a las amenazas que se presentan en su entorno. Esta teoría explica que la creación de las ventajas competitivas se logra gracias a los recursos y capacidades que se desarrollan dentro de la organización; los primeros se constituyen de aquellos activos tangibles e intangibles vinculados a ella en un cierto periodo de tiempo; y las capacidades son la forma en la que la empresa realiza sus actividades, dependiendo de los recursos con los que dispone (Grant 1991; Wernerfelt 1984).

Varios estudios han confirmado que lo que realmente es necesario para que la empresa alcance y mantenga una ventaja competitiva, se deriva de sus recursos intangibles y sus capacidades, porque se basan en datos no codificados y conocimiento tácito, lo que dificulta que se imiten, pues se requiere un proceso lento de desarrollo (Peteraf 1993; Barney 1991).

En las PYMES, las inversiones en recursos intangibles y la creación de capacidades se realiza con mayor dificultad a diferencia de lo que ocurre en las grandes empresas, porque para que las primeras puedan obtener una ventaja competitiva de sus recursos y capacidades, deben aumentar su escala de producción y ser más eficientes en el uso de sus recursos (Pil y Holweg, 2003). Por ello, es necesario indagar si la forma en que se administran los recursos y las capacidades en las PYMES, se ve influida por la orientación estratégica y por las características del dueñoadministrador. 
Los estudiosos en las áreas administrativas se han dado a la tarea de caracterizar distintos perfiles en los empresarios, para explicar su comportamiento presente y predecir su comportamiento futuro. El trabajo más influyente es quizá el de Miles y Snow (1978), quienes definen cuatro arquetipos estratégicos a partir de su posición sobre innovación, monitoreo de mercado, estrategia, operación, riesgo y las habilidades que han desarrollado para adaptarse a los cambios del entorno.

Sobre el trabajo original de Miles y Snow, DeSarbo, Di Benedetto, Song y Sinha (2005), determinaron que el arquetipo estratégico se modifica según las circunstancias que están viviendo como parte de la adaptación constante y necesaria, que deben realizar para seguir manteniéndose en el mercado (Romanelli y Tushman, 1994).

Los arquetipos estratégicos identificados por Miles y Snow (1978) y complementados por DeSarbo, et al., (2005), se definen por su manera de actuar a partir de las actividades que realizan de manera permanente. Todos ellos tienen estrategias diferentes que desarrollan con el objetivo de maximizar utilidades. Sus principales características se resumen a continuación.

- Reactor: Las empresas regidas por este arquetipo, no cuentan con ningún tipo de estrategia para el desarrollo de sus funciones, por lo que su única opción es reaccionar ante los cambios y emergencias que surgen en el entorno, lo que implica que no puedan controlar su entorno interno y externo, por lo que presentan dificultades para hacer frente a la competencia.

- Defensor: Su principal característica es que se preocupa en resolver los problemas de ingeniería de sus procesos. De igual manera reducen su mercado, ya que prefieren que sea estable, limitado y conocido. Las empresas con este arquetipo se pueden catalogar como expertas en la fabricación de los bienes y servicios que ofrecen. Su producción, inventario, su proceso de abastecimiento y de distribución, están muy controlados. Sin embargo, el tener un control tan rígido, provoca carencia de movimiento o de adaptación para cambios que a veces imponen la sociedad o los mismos clientes.

- Analista: Este arquetipo intenta equilibrar el riesgo entre la provisión de los productos o servicios y una amplia gama de utilidades. Una de sus características más sobresalientes es que siempre van a buscar otros productos y servicios para lanzar al mercado, pero la inversión que tienen que realizar proviene generalmente de los mismos productos y servicios que serán lanzados, por lo que el riesgo que asumen es mínimo.

- Prospector: Utiliza estrategias exploradoras y busca constantemente mejores oportunidades en el mercado. Disfruta realizar innovaciones anticipándose casi siempre, a las necesidades de sus clientes. Trabaja constantemente con el departamento de 
marketing para darse a conocer y de esta forma, aumentar su volumen de bienes o servicios que ofrecen al mercado. De la misma manera, buscan incrementar su cartera de clientes, pero se diversifican demasiado y no se hacen expertos en nada; sin embargo, una vez que conocen el entorno, logran sostenerse y permanecer.

Dependiendo de la orientación estratégica adoptada, la empresa puede enfatizar más o menos aspectos como la la innovación, el monitoreo de mercado, la estrategia, la operación, su posición ante el riesgo y su capacidad de adaptación. En la tabla 1 se muestra la posición de cada arquetipo con respecto a estas premisas.

Tabla 1:

Posturas de los arquetipos estratégicos

\begin{tabular}{|c|c|c|c|c|c|c|}
\hline Arquetipo & Innov aciones & $\begin{array}{l}\text { Monitoreo de } \\
\text { Mercado }\end{array}$ & Estrategia & Operación & Riesgo & Adaptación \\
\hline Reactor & Mínimas & $\begin{array}{l}\text { Minima, sólo } \\
\text { cuando existe } \\
\text { un evidente } \\
\text { riesgo }\end{array}$ & $\begin{array}{l}\text { Reaccionan } \\
\text { ante cambios } \\
\text { y } \\
\text { emergencias } \\
\text { que surgen } \\
\text { en el entorno }\end{array}$ & $\begin{array}{l}\text { Resuelven } \\
\text { problemas } \\
\text { de } \\
\text { operación } \\
\text { que se } \\
\text { presentan } \\
\text { a diario }\end{array}$ & $\begin{array}{l}\text { Se mueven } \\
\text { para } \\
\text { mantenerlo } \\
\text { al minimo }\end{array}$ & $\begin{array}{l}\text { Sólo en } \\
\text { caso de } \\
\text { evidente } \\
\text { riesgo }\end{array}$ \\
\hline Defensor & $\begin{array}{l}\text { En procesos, } \\
\text { para ser más } \\
\text { eficientes }\end{array}$ & $\begin{array}{l}\text { Monitoreo para } \\
\text { cerciorarse de } \\
\text { que el mercado } \\
\text { se mantiene } \\
\text { estable, } \\
\text { limitado y } \\
\text { conocido }\end{array}$ & $\begin{array}{l}\text { Especialistas. } \\
\text { Expertos en la } \\
\text { fabricación } \\
\text { de bienes y } \\
\text { servicios que } \\
\text { ofrecen }\end{array}$ & $\begin{array}{l}\text { Resuelve } \\
\text { problemas } \\
\text { de } \\
\text { ingeneria } \\
\text { en sus } \\
\text { procesos }\end{array}$ & $\begin{array}{l}\text { Cambios } \\
\text { en sus } \\
\text { reducidos } \\
\text { pero } \\
\text { estables } \\
\text { clientes }\end{array}$ & Lento \\
\hline Analista & $\begin{array}{l}\text { En procesos, } \\
\text { y en } \\
\text { productos, } \\
\text { después de } \\
\text { un cuidadoso } \\
\text { análisis }\end{array}$ & $\begin{array}{l}\text { Regularmente, } \\
\text { buscando } \\
\text { oportunidades } \\
\text { de mercado, } \\
\text { después de un } \\
\text { análisis } \\
\text { detallado }\end{array}$ & $\begin{array}{l}\text { Equilibra el } \\
\text { riesgo con la } \\
\text { provisión de } \\
\text { los productos } \\
\text { y su utilidad }\end{array}$ & $\begin{array}{l}\text { Equilibrada } \\
\text { con lo que } \\
\text { ya conoce } \\
\text { y lo que } \\
\text { analiza } \\
\text { que puede } \\
\text { funcionar }\end{array}$ & $\begin{array}{l}\text { Riesgo en } \\
\text { procesos, } \\
\text { productos } \\
\text { y } \\
\text { mercados } \\
\text { probados. } \\
\text { Son } \\
\text { seguidores }\end{array}$ & Alta \\
\hline Prospector & $\begin{array}{l}\text { En } \\
\text { productos, } \\
\text { anticipándose } \\
\text { a las } \\
\text { necesidades } \\
\text { de sus } \\
\text { clientes }\end{array}$ & $\begin{array}{l}\text { Buscan } \\
\text { constantemente } \\
\text { mejores } \\
\text { oportunidades. } \\
\text { Busca } \\
\text { incrementar } \\
\text { clientes }\end{array}$ & $\begin{array}{l}\text { Exploradoras } \\
\text { y } \\
\text { diversificadas }\end{array}$ & $\begin{array}{l}\text { Constante } \\
\text { y } \\
\text { cambiante }\end{array}$ & $\begin{array}{l}\text { No se } \\
\text { hacen } \\
\text { expertos } \\
\text { en nada }\end{array}$ & Muy alta \\
\hline
\end{tabular}

Fuente: Elaboración propia con base a Miles y Snow (1978) y DeSarbo, et al., (2005).

La tipología de Miles y Snow (1978) sobre los arquetipos estratégicos, ha tenido un gran impacto dentro de los estudios administrativos. Sin embargo, la mayoría de las investigaciones existentes, se han limitado al contexto de las grandes empresas y, con muy pocas excepciones, este tipo de análisis se ha realizado en el contexto de las PYMES, en donde el papel del que dirige la organización es fundamental, por lo que se hace necesario analizar sus características y su relación con la innovación y el desempeño. 
Aunque existen diversos estudios que han analizado las características del dueño administrador en PYMES, no se encontraron investigaciones que analicen las diferencias en gestión entre los dueños y administradores, porque en este tipo de unidades de negocio, se da por hecho que ambos son la misma persona.

Sin embargo, un punto de referencia es el estudio de Stewart y Roth (2007), que identifica diferencias claras entre dueños y administradores, encontrando que los primeros mantienen más altos niveles de motivación por un periodo más largo de tiempo, pues su empresa es parte de su realización como seres humanos, afectando esto también en su forma de gestionar, ya que fácilmente pierden la objetividad de los asuntos por cuestiones personales. Marcketti, et al. (2006), explican que los dueños de las PYMES comienzan a emprender en áreas que reflejan su propia experiencia e intereses personales; mientras que los administradores gestionan sobre lo que los dueños han logrado hacer. Los dueños de las PYMES por consiguiente, mantienen un punto de vista un tanto subjetivo sobre los alcances y limitaciones de sus empresas (Arenius y Minniti, 2005; Grilo y Thurik, 2008), mientras que los administradores pueden desarrollar un punto de vista más impersonal, por ser ajenos a su origen.

En cuanto al nivel de estudio, Gray (2006) encontró que los dueñosadministradores que tienen estudios superiores y que son adultos jóvenes, desarrollan mayores capacidades para la innovación, pues están más abiertos al aprendizaje permanente.

El área de conocimiento en el que se forman los dueñosadministradores, influye en el sector en el que se involucran más y, en el caso de los dueños, es en donde deciden emprender (Marcketti et al., 2006).

Los años de experiencia que los dueños y administradores van acumulando, es sin duda un factor que afecta en sus habilidades para innovar, pues los más jovenes estarán más abiertos a realizar cambios y a arriesgarse a desarrollar nuevos mercados, aunque los más experimentados conocen los riesgos y calculan con mayor precisión los costos en los que se incurren por la puesta en marcha de esas innovaciones.

\section{Innovación y su relación con dueños-administradores}

La innovación puede observarse en los procesos tecnológicos, las estructuras organizativas, sus sistemas de administración y los programas que utilizan. Todos estos aspectos pueden afectar en el desempeño de la organización. Pero la innovación no siempre tiene un efecto positivo en el corto plazo sobre el desempeño de las PYMES. Existen muchos otros factores que interactúan para el resultado final, como el contexto y el ciclo de vida del producto (Rosenbusch, Brinckmann y Bausch, 2011), así como la manera de utilizar la información que brinda el entorno. 
El nivel de innovación constituye un elemento básico para lograr la competitividad y de acuerdo a Miles y Snow (1978), las estrategias son diferentes dependiendo de las innovaciones que desarrollan los dueños administradores respecto a sus competidores. Los arquetipos prospectores prestan más atención en la innovación de sus productos o de su mercado como un medio para obtener una ventaja competitiva, mientras que los arquetipos defensores se centran más en mejorar su eficiencia, auque ello implique mantener una posición tecnológica más débil.

Los estudios existentes del tema han corroborado algunos puntos, Por ejemplo, McDaniel y Kolari (1987) afirman que el grado de innovación de los prospectoras es significativamente mayor que el que tienen los defensores, aunque tal grado de innovación es similar entre los prospectores y los analistas.

Eisenhardt y Schoonhoven (1996) argumentaron que el grado de innovación es el elemento estratégico fundamental que determina la necesidad de cooperación y descubrieron que las PYMES con estrategias más proactivas e innovadoras, buscarán siempre un mayor número de acuerdos de cooperación que las empresas con estrategias más conservadoras como las que tienen un arquetipo analista o defensor, debido a la mayor necesidad de recursos para mantener su nivel de tecnología e innovación.

Las empresas con una orientación prospectora implementan un gran número de prácticas, lo que lleva a una mayor flexibilidad, debido a la innovación constante que persiguen. Las empresas consideradas como defensoras, son menos flexibles porque un mínimo cambio podría afectar su eficiencia y su objetivo de minimización de costos (Conant, Mokwa, Varadarajan 1990).

En el contexto de las PYMES, Camison (1997) también encontró que las más innovadoras son las prospectoras, quienes adoptan prácticas más flexibles que las analizadoras y defensoras. Sin embargo, a corto y mediano plazo, las prospectoras no siempre son las que tienen un mejor desempeño.

\section{Relación entre desempeño de las PYMES y caracteristicas del dueño- administrador}

El desempeño de las PYMES no sólo está dado por sus recursos y capacidades y el contexto en el que se desenvuelven, también está fuertemente influenciado por las características de quien dirige a estas unidades económicas (Hansen y Hamilton, 2011). Al respecto, Doty, Glick y Huber (1993) concluyeron que la tipología de arquetipos estratégicos de Miles y Snow es un poderoso predictor de la eficiencia del negocio y, por supuesto, del desempeño. Por ello, es un referente para este trabajo.

Miles y Snow (1978) y más recientemente Conant, Mokwa y Varadarajan (1990) y Wright et al. (1991) explican que los prospectores, analistas y defensores tienen la oportunidad de ser igualmente exitosos en 
el desarrollo de sus actividades y que, en cualquier caso, estos tres tipos estratégicos funcionarán mejor que los reactores.

En el contexto de las PYMES, Camison (1997, p. 413) examinó las relaciones entre la orientación estratégica de las PYMES y su nivel de desempeño. Utilizando tres medidas de rendimiento: rentabilidad, productividad y participación de mercado, el autor descubrió que las organizaciones más rentables y productivas son las que tienen comportamientos estratégicos más proactivos. AragónSánchez y Sánchez-Marín (2005) encontraron que las PYMES con arquetipos prospectores, tienen mejores resultados en el desempeño que las defensoras; sin embargo, el estudio lo realizaron sobre distintos sectores, por lo que los resultados no pueden generalizarse.

Además de los estudios anteriores, existen otros que explican la vinculación con otras características de los dueños y el desempeño. Richbell, Brookes, Brewster y Wood (2006) y Delmar y Wiklund (2008) encontraron una relación fuerte y significativa entre la educación del dueño, su área de experiencia y el desempeño de la PYME. Barbero, Casillas y Feldman (2011), analizaron las capacidades del dueñoadministrador y los resultados obtenidos por las PYMES, descubriendo que los que tienen mayor inclinación a las áreas de mercadotecnia y finanzas, tienen mayores posibilidades de expansión de mercados y de incentivar las innovaciones en productos.

Sin embargo, en un estudio con PYMES de distintos sectores, Blackburn, Hart y Wainwrigth (2013), encontraron que las características propias de las PYMES y su sector son más influyentes en su desempeño, que las características del dueño-administrador y su arquetipo estratégico, razón por la cual esta investigación se concentró al análisis particular de PYMES metalmecánicas de la industria automotriz, que tienen sus propias características y problemas.

\section{Pymes metalmecánicas de la industria automotriz}

En nuestros días, el desarrollo de la industria automotriz en México se ha incrementado exponencialmente, pues la mayoría de los grandes corporativos de automóviles en el mundo, han vislumbrado grandes oportunidades por los abundantes recursos disponibles, mano de obra calificada y por supuesto, la ubicación geográfica (Vicencio Miranda, 2007). Por tal motivo, hoy en día esta industria lidera las inversiones que empresas extranjeras hacen en el campo de la manufactura mexicana. Según datos del INEGI y de la Asociación Mexicana de la Industria Automotriz (2016), la industria manufacturera aporta el 3.5\% del PIB nacional y $16.9 \%$ del PIB manufacturero, alcanzando el octavo lugar como productor de vehículos en el mundo y el cuarto como exportador mundial.

Sin embargo, en la última década, la industria automotriz en Latinoamérica se ha visto opacada porque los fabricantes de automóviles asiáticos han incrementado sus exportaciones hacia Estados Unidos (Álvarez Medina, 2016; Avendano, Melguizo y Miner, 2017). 
Esto ha afectado considerablemente a las PYMES metalmecánicas, que han sido un eslabón importante en la cadena de proveeduría de la industria automotriz, porque proveen de maquinarias e insumos a la mayoría de las actividades económicas vinculadas al sector, constituyendo la mayor parte de los proveedores nacionales dentro de los eslabones Tier 1,2 y 3 de la cadena productiva (Bautista y Peralta, 2017).

Se estima que existen más de 550 proveedores nacionales de primer nivel (Tier 1) que a su vez, adquieren suministros para su producción a empresas más pequeñas (Tier 2 y Tier 3) que se estima son alrededor de 400 (Covarrubias Valdenebro, 2014).

Ante los constantes cambios (Vicencio Miranda, 2007) y demandas de la industria automotriz (Álvarez Medina, 2016), las PYMES metalmecánicas se han esforzado para desarrollar nuevas competencias tecnológicas y organizativas, pero parecen no ser suficientes. Las PYMES de segundo y tercer nivel deben cumplir con parámetros establecidos por las grandes empresas automotrices, y, al mismo tiempo, deben re-pensar en abordar otros mercados, contar con otros clientes y tener una gama más amplia de productos que puedan apoyarlos a sobrevivir (González y Luna, 2016), a partir de prácticas innovadoras en pro de un mejor desempeño de la organización (Damanpour y Scneider, 2006). Para la mayoría de los pequeños empresarios el reto es mayúsculo, porque están mayormente ocupados en su desempeño a corto plazo (Baptista Nunes, Annansingh, Eaglestone y Wakefield, 2006).

En este sentido, existen diversos estudios que explican la relación estrecha entre la visión y análisis del mercado que realizan los directivos y la innovación en productos, mercados y procesos (Becker y Peters, 2000; Cockburn y Henderson, 1998; Cohen y Levinthal, 1990; Nieto y Quevedo, 2005; Veugelers, 1997). Sin embargo, la mayoría de estos estudios se han realizado en grandes empresas, las cuales poseen estrategias más definidas que las PYMES y en las que la definición de éstas, no se concentra en una sola persona.

Por tal razón y con el objetivo de analizar la relación entre las características del dueño-administrador de las PYMES metalmecánicas de la industria automotriz del Valle de Toluca con la innovación y desempeño, se entrevistó a una muestra de dueños y administradores de éstas, considerando su arquetipo estratégico, su papel como dueño o administrador, el nivel de estudios y la experiencia en el ramo, para generar evidencias que soportan estudios anteriores y desarrollar estrategías que les ayuden a enfrentar el entorno cambiante que se avecina.

\section{Metodología}

El desarrollo de la industria automotriz en México se ha incrementado fuertemente en los últimos años, pues muchos de los grandes corporativos de automóviles en el mundo, han vislumbrado grandes oportunidades al instalarse en México (Vicencio Miranda, 2007). Sin embargo, para las PYMES pertenecientes a ese sector, se avecina un entorno complejo dado sobre todo por la incertidumbre en las reglas de operación de la 
última modificación al Tratado de Libre Comercio con Estados Unidos y Canadá (Estañol et al., 2016) y por la fuerza que están adquiriendo los fabricantes de automóviles asiáticos (Álvarez Medina, 2016).

Considerando que en las PYMES el dueño-administrador adapta las estrategias de acuerdo a las circunstancias que va marcando el entorno (Apak y Atay, 2014; Bagnoli y Vedovato, 2012), se hace necesario realizar investigaciones sobre las características del dueño-administrador y su relación con la innovación y desempeño, para generar estrategias adecuadas a sus propias circunstancias.

El objetivo de esta investigación fue analizar la relación que existe entre las características del dueño-administrador y la orientación estratégica sobre la innovación y desempeño de las PYMES metalmecánicas del Valle de Toluca. Los objetivos específicos fueron:

- Determinar el arquetipo estratégico que existe en el dueño o administrador de la PYME del sector metalmecánico en el Valle de Toluca.

- Determinar las características del dueño o administrador de la PYME metalmecánica del Valle de Toluca que tienen mayor relación con la innovación y el desempeño.

Utilizando como instrumento de recolección de datos una traducción del original de DeSarbo et al., (2005) para clasificar el arquetipo estratégico, de Subramaniam y Youndt (2005) para la escala de innovación y de Schreiner, Kale y Corsten (2009) para medir el desempeño percibido, se encuestaron a 51 pequeños y medianos empresarios de los giros que conforman el sector metalmecánico (INEGI y AMIA, 2016) dentro del Valle de Toluca ${ }^{[1]} .27$ de ellos son dueños de la PYME y 24 fungen como administradores.

Para medir las variables de estudio (innovación y desempeño) se utilizó una escala de Likert, calificando la percepción de las acciones realizadas y los resultados logrados comparándose con su competencia, en donde 1 significó "mucho peor que la competencia" y el 7 "mucho mejor que la competencia".

Para verificar la confiabilidad del instrumento, se calculó el Alfa de Cronbach, determinando un parámetro aceptable en las escalas, ya que se logró una consistencia interna mayor a 0.700 , lo razonable para este tipo de estudios (Hair, Prentice y Cano, 1999).

La investigación fue de corte exploratoria descriptiva y aplicada: a partir de investigaciones sobre el tema, se desarrolló una investigación cuantitativa realizando cálculos estadísticos que sirvieron de base para la discusión de los resultados.

Debido a que se recogen las percepciones de los pequeños y medianos empresarios y administradores, esta investigación es no experimental, ya que se trata de observar el fenómeno sin realizar ninguna alteración a la realidad. Por el periodo en que es efectuada es una investigación transversal, recolectando los datos en un tiempo determinado (febrero de 2017), al igual que el fenómeno estudiado. 
Se recurrió a una muestra no probabilística (sujetos voluntarios); las unidades son los directivos y dueños de PYMES metalmecánicas del Valle de Toluca.

Las hipótesis en las que se basó este trabajo:

$\mathrm{H}_{1}=$ Existe una relación fuerte y significativa entre alguna característica del dueño o administrador con el desempeño y la innovación de la PYME metalmecánica del Valle de Toluca.

$\mathrm{H}_{0}=$ No existe ninguna relación fuerte y significativa entre alguna característica del dueño o administrador con el desempeño y la innovación de la PYME metalmecánica del Valle de Toluca.

\section{Resultados y discusión}

El Alfa de Cronbach (tabla 2) determinó un parámetro aceptable de confiabilidad en las escalas, ya que se logró una consistencia interna por arriba del 0.85 , mayor a lo razonable para este tipo de estudios (Hair, Prentice y Cano, 1999). Por ende, se respetaron las escalas originales con el número de preguntas de los instrumentos originales utilizados para esta investigación.

Tabla 2:

Alfa de Crobanch para las variables de estudio

\begin{tabular}{lll}
\hline Variable & $\begin{array}{c}\text { Preguntas } \\
\text { en la } \\
\text { escala } \\
\text { original }\end{array}$ & $\begin{array}{c}\text { Alfa de } \\
\text { Cronbach }\end{array}$ \\
\hline Innovación & 6 & 0.872 \\
\hline Desempeño & 4 & 0.856 \\
\hline
\end{tabular}

Fuente: Elaboración propia con cálculos de SPSS 23.0.

Los resultados determinan que los pequeños empresarios entrevistados de la industria metalmecánica de la industria automotriz del Valle de Toluca, se comportan con un arquetipo estratégico analista o defensor (tabla 3).

Tabla 3:

Clasificación de empresarios por arquetipo estratégico

\begin{tabular}{lll}
\hline Arquetipo & Entrevistados & Porcentaje \\
\hline Analista & 24 & $47 \%$ \\
\hline Defensor & 27 & $53 \%$ \\
\hline Total & 51 & $100 \%$ \\
\hline
\end{tabular}

Fuente: Elaboración propia.

Del total de los entrevistados (tanto dueños como administradores), se determinó que 24 tienen un arquetipo estratégico analista (que representan el $47 \%$ de la población), quienes afirmaron tener pocos productos y realizar pocas innovaciones después de un cuidadoso análisis 
del riesgo, concentrando sus esfuerzos en satisfacer la demanda de los escasos pero selectos clientes que tienen de forma recurrente.

En cuanto a los 27 encuestados restantes, se determinó que tienen un arquetipo defensor (el 53\% de la población). Ellos perciben que sus productos son estables porque el mercado los ha definido. Ofrecen pocos productos pero selectos y de alta calidad. Afirmaron dedicar mayores recursos en defender los mercados que ya cubren, considerando que están preparados para las amenazas que pueden surgir en contra del negocio.

A partir de los dos grupos de arquetipos estratégicos y el cálculo de las variables compuestas, se obtuvieron medias y desviaciones estándar. En la tabla 4 se observan los descriptivos para cada grupo, mostrando que ambos consideran estar por arriba del valor medio de las escalas de innovación y desempeño. Se observa un ligero valor superior en el grupo de los analistas sobre los defensores en ambas variables; sin embargo, existe una mayor dispersión de los datos en innovación del grupo de los analistas comparando con los defensores.

Tabla 4:

Estadísticos descriptivos por grupo

\begin{tabular}{llllllll}
\hline & \multicolumn{3}{c}{ General } & \multicolumn{2}{c}{ Analistas } & \multicolumn{2}{c}{ Defensores } \\
\cline { 2 - 8 } & $\begin{array}{l}\text { Valor } \\
\begin{array}{l}\text { medio } \\
\text { de la } \\
\text { escala }\end{array}\end{array}$ & Media & $\begin{array}{l}\text { Desviación } \\
\text { estándar }\end{array}$ & Media & $\begin{array}{l}\text { Desviación } \\
\text { estándar }\end{array}$ & Media & $\begin{array}{l}\text { Desviación } \\
\text { estándar }\end{array}$ \\
\hline Innovación & 3.5 & 4.38 & 1.650 & 4.72 & 1.691 & 4.07 & 1.583 \\
\hline Desempeño & 3.5 & 4.75 & 1.489 & 5.03 & 1.458 & 4.51 & 1.501 \\
\hline
\end{tabular}

Fuente: Elaboración propia con cálculos de SPSS 23.0.

Se utilizó la prueba t de student (tabla 5), determinando que no existe diferencia significativa ente las medias de innovación y desempeño entre los grupos de analistas y defensores. Por ello, no se puede afirmar que el arquetipo estratégico de los entrevistados y que forman parte de la muestra de esta investigación, sea un factor determinante en la innovación y desempeño de la PYME.

Tabla 5:

Prueba t de student para muestras independientes

\begin{tabular}{lll}
\hline Variable & $\begin{array}{l}\text { P-Valor } \\
\text { (Sig } \\
\text { bilateral) }\end{array}$ & $\begin{array}{l}\text { Aceptación o rechazo de H1 a un nivel } \\
\text { de significancia a }=0.05\end{array}$ \\
\hline Innovación & 0.196 & $\begin{array}{l}\text { No existe diferencia significativa entre } \\
\text { la media de innovación de analistas y } \\
\text { defensores }\end{array}$ \\
\hline Desempeño & 0.653 & $\begin{array}{l}\text { No existe diferencia significativa entre } \\
\text { la media de desempeño de analistas y } \\
\text { defensores }\end{array}$ \\
\hline
\end{tabular}

Fuente: Elaboración propia con cálculos de SPSS 23.0. 
Con el fin de considerar las características del dueño-administrador que mayor relación tienen con las variables de innovación y desempeño, se analizó la correlación bivariada entre las variables del estudio a través de la correlación de Spearman (tabla 6).

Tabla 6:

Correlación de Spearman para las variables de estudio

\begin{tabular}{|c|c|c|c|c|c|c|}
\hline & Desempeño & Dueño-administrador & $\begin{array}{l}\text { Nivel } \\
\text { educativo }\end{array}$ & $\begin{array}{l}\text { Area } \\
\text { Admon }\end{array}$ & Experiencia & $\begin{array}{l}\text { Arquetipo } \\
\text { dominante }\end{array}$ \\
\hline Innovación & $673^{* *}$ & $-.493^{* *}$ & .220 & -.067 & .272 & .094 \\
\hline Desempeño & 1 & -.216 & .068 & .041 & $410^{* *}$ & -.005 \\
\hline Dueño-administrador & & 1 & .076 & .021 & -.240 & .096 \\
\hline Nivel educativo & & & 1 & $-.339^{*}$ & -.098 & .220 \\
\hline Area Admon & & & & 1 & .097 & .045 \\
\hline Experiencia & & & & & 1 & .000 \\
\hline
\end{tabular}

Fuente: Elaboración propia con cálculos de SPSS 23.0.

${ }^{* *}$ La correlación es significativa en el nivel 0,01 (bilateral).

${ }^{*}$ La correlación es significativa en el nivel 0,05 (bilateral).

Se encontraron correlaciones significativas entre la innovación con el estatus de dueño o administrador y el desempeño con la experiencia. Considerando este hallazgo y lo que el estudio de Marcketti, et al. (2006) encontraron sobre las diferencias de gestión entre dueños y administradores, se decidió eron como los siguientes grupos de estudio los dueños y los administradores.

En la tabla 7 se presentan los descriptivos para ambos grupos, mostrando que los dueños consideran estar por arriba del valor medio de las escalas de innovación y desempeño. Por su parte, los administradores consideran estar en la media del valor de la escala de innovación y perciben estar por arriba del valor medio de la escala de desempeño. Se observa un valor superior en el grupo de los dueños sobre los administradores en ambas variables, con una diferencia mayor en la innovación entre dueños y administradores. Además, se muestra una mayor dispersión de los datos en la innovación del grupo de los administradores.

Tabla 7:

Estadísticos descriptivos por grupo de dueños y administadores

\begin{tabular}{llllllll}
\hline & \multicolumn{3}{c}{ General } & Dueño (27) & \multicolumn{2}{c}{$\begin{array}{l}\text { Administrador } \\
\text { (24) }\end{array}$} \\
\cline { 2 - 8 } & $\begin{array}{l}\text { Valor } \\
\begin{array}{l}\text { medio } \\
\text { de la } \\
\text { escala }\end{array}\end{array}$ & & $\begin{array}{l}\text { Desviación } \\
\text { estándar }\end{array}$ & Media & $\begin{array}{l}\text { Desviación } \\
\text { estándar }\end{array}$ & Media & $\begin{array}{l}\text { Desviación } \\
\text { estándar }\end{array}$ \\
\hline Innovación & 3.5 & 4.38 & 1.650 & 5.14 & 1.158 & 3.52 & 1.721 \\
\hline Desempeño & 3.5 & 4.75 & 1.489 & 5.06 & 1.368 & 4.42 & 1.575 \\
\hline
\end{tabular}


Para verificar si las diferencias encontradas en los descriptivos son significativas entre los grupos de dueños y administradores, nuevamente se utilizó la prueba t de student (tabla 8), determinando que en las medias de innovación, sí existen diferencias significativas entre los grupos de dueños y administradores, pero no en las medias de desempeño.

Tabla 8:

Prueba t de student para muestras independientes

\begin{tabular}{lll}
\hline Variable & $\begin{array}{l}\text { P-Valor } \\
\text { (sig } \\
\text { bilateral) }\end{array}$ & $\begin{array}{l}\text { Aceptación o rechazo de H1 a un nivel } \\
\text { de significancia a =0.05 }\end{array}$ \\
\hline Innovación & 0.046 & $\begin{array}{l}\text { Existe diferencia significativa entre la } \\
\text { media de innovación de dueños y } \\
\text { administradores }\end{array}$ \\
\hline Desempeño & 0.405 & $\begin{array}{l}\text { No existe diferencia significativa entre } \\
\text { la media de desempeño de dueños y } \\
\text { administradores }\end{array}$ \\
\hline
\end{tabular}

Fuente: Elaboración propia con cálculos de SPSS 23.0.

A partir de estos hallazgos y con el fin de realizar un análisis más profundo sobre las diferencias encontradas, se calculó la correlación de Spearman para los grupos de dueños y administradores (tabla 9) con las características de los dueños-administradores consideradas para esta investigación.

Tabla 9:

Correlación de Spearman para dueños y administradores

Fuente: Elaboración propia con cálculos de SPSS 23.0.

${ }^{* *}$ La correlación es significativa en el nivel 0,01 (bilateral).

${ }^{*}$ La correlación es significativa en el nivel 0,05 (bilateral).

De acuerdo a los cálculos anteriores, se observa que en el grupo de los dueños, el nivel educativo y el arquetipo estratégico son factores que pueden influir en la innovación de las PYMES, mientras que la experiencia es un factor que tiene relación con en desempeño. Por otro lado, en el grupo de administradores, el nivel educativo y la experiencia también son factores que influyen en la innovación y desempeño respectivamente.

Considerando estos resultados, se construyó una tabla cruzada (tabla 10) que muestra los puntajes de innovación y desempeño definidos por los propios entrevistados a prtir del cuestionario aplicado, analizando la combinación de los factores que presentaron mayor relación con la innovación y desempeño, es decir, nivel educativo, experiencia y arquetipo dominante.

En la muestra del estudio, de los 27 dueños de las PYMES, 16 se identificaron con un arquetipo estratégico defensor y 11 analistas. Por otra parte, en el grupo de administradores conformado por 24 entrevistados, se identificaron 13 con arquetipo estratégico defensor y 
11 analistas. En ambos grupos (dueños y administradores) predomina el arquetipo estratégico defensor.

En cuanto a nivel educativo, la mayoría de los que están al frente de la organización cuentan con una licenciatura. Solo en el caso de los dueños con arquetipo analista, se encontró que predominan los que cuentan con preparación técnica.

Sobre los años de experiencia, en el grupo de los dueños predominan los que tienen entre 5 y 20 años en el puesto. En tanto, en el grupo de los administradores predominan los que tienen entre 2 y 10 años en la organización.

Para el grupo de los dueños en cuanto a los resultados de innovación, destaca el hallazgo que en el grupo de dueños con arquetipo defensor, todos perciben estar por arriba de la media de la escala (la calificación mínima fue 4.0), mientras que en el grupo de los dueños con arquetipo analista, se observa una mayor dispersión entre los datos, pues la calificación mínima fue de 3.83 y la máxima es de 7.0. Los mejores resultados del grupo de los dueños, se dieron entre los que tienen más de 20 años de experiencia y que han cursado una licenciatura o posgrado.

Analizando los resultados del grupo de los dueños para desempeño, el resultado más deficiente se presenta en los que tienen licenciatura, arquetipo estratégico defensor y cuentan con dos o tres años de experiencia en la organización. Por otra parte, el mejor resultado de desempeño lo obtuvo el entrevistado que tiene entre 4 a 5 años de experiencia, tiene un arquetipo analista y cursó una carrera técnica.

\section{Tabla 10:}

resultados de innovación y desempeño por grupos de dueños y administradores, arquetipos, educación formal y experiencia

\begin{tabular}{|c|c|c|c|c|c|c|c|c|c|c|c|c|c|c|c|c|}
\hline \multirow{3}{*}{ Dueño } & \multicolumn{8}{|c|}{ Defensor (16) } & \multicolumn{8}{|c|}{ Analista (11) } \\
\hline & \multicolumn{2}{|c|}{$\begin{array}{c}\text { Sin educación } \\
\text { superior }(0)\end{array}$} & \multicolumn{2}{|c|}{ Técnico (4) } & \multicolumn{2}{|c|}{$\begin{array}{l}\text { Licenciatura } \\
(9)\end{array}$} & \multicolumn{2}{|c|}{ Posgrado (3) } & \multicolumn{2}{|c|}{$\begin{array}{c}\text { Sin educación } \\
\text { superior (1) }\end{array}$} & \multicolumn{2}{|c|}{ Técnico (5) } & \multicolumn{2}{|c|}{$\begin{array}{c}\text { Licenciatura } \\
\text { (4) }\end{array}$} & \multicolumn{2}{|c|}{ Posgrado (1) } \\
\hline & $\begin{array}{l}\text { Inno- } \\
\text { vación }\end{array}$ & $\begin{array}{c}\text { Desem- } \\
\text { peño }\end{array}$ & $\begin{array}{l}\text { Inno- } \\
\text { vación }\end{array}$ & $\begin{array}{c}\text { Desem- } \\
\text { peño }\end{array}$ & $\begin{array}{l}\text { Inno- } \\
\text { vación }\end{array}$ & $\begin{array}{c}\text { Desem- } \\
\text { peño }\end{array}$ & $\begin{array}{l}\text { Inno- } \\
\text { vación }\end{array}$ & $\begin{array}{l}\text { Desem- } \\
\text { peño }\end{array}$ & $\begin{array}{l}\text { Inno- } \\
\text { vación }\end{array}$ & $\begin{array}{c}\text { Desem- } \\
\text { peño }\end{array}$ & $\begin{array}{l}\text { Inno- } \\
\text { vación }\end{array}$ & $\begin{array}{c}\text { Desem- } \\
\text { peño }\end{array}$ & $\begin{array}{l}\text { Inno- } \\
\text { vación }\end{array}$ & $\begin{array}{c}\text { Desem- } \\
\text { peño }\end{array}$ & $\begin{array}{l}\text { Inno- } \\
\text { vación }\end{array}$ & $\begin{array}{l}\text { Desem- } \\
\text { peño }\end{array}$ \\
\hline Menos de 2 años de experiencia & - & - & - & - & - & - & 4.00 & 2.00 & - & - & - & - & - & - & - & - \\
\hline De 2 a 3 años de experiencia & - & - & 4.00 & 4.75 & 4.00 & 1.00 & - & - & - & - & - & - & - & - & - & - \\
\hline De 4 a 5 años de experiencia & - & - & 5.17 & 5.25 & 5.25 & 6.00 & 4.33 & 4.25 & 3.83 & 3.50 & 7.00 & 7.00 & 6.17 & 5.38 & - & - \\
\hline De 5 a 20 años de experiencia & - & - & - & - & 5.58 & 5.31 & 5.00 & 4.50 & - & - & 3.94 & 4.92 & 6.67 & 6.25 & - & - \\
\hline Más de 20 años de experiencia & - & - & 4.17 & 4.00 & 4.75 & 5.75 & - & - & - & - & 6.00 & 6.50 & 7.00 & 6.25 & 7.00 & 6.25 \\
\hline \multirow{3}{*}{ Administrador } & \multicolumn{8}{|c|}{ Defensor (13) } & \multicolumn{8}{|c|}{ Analista (11) } \\
\hline & \multicolumn{2}{|c|}{$\begin{array}{l}\text { Sin educación } \\
\text { superior (3) }\end{array}$} & \multicolumn{2}{|c|}{ Técnico (2) } & \multicolumn{2}{|c|}{$\begin{array}{c}\text { Licenciatura } \\
\text { (4) }\end{array}$} & \multicolumn{2}{|c|}{ Posgrado (4) } & \multicolumn{2}{|c|}{$\begin{array}{l}\text { Sin educación } \\
\text { superior (1) }\end{array}$} & \multicolumn{2}{|c|}{ Técnico (0) } & \multicolumn{2}{|c|}{$\begin{array}{l}\text { Licenciatura } \\
\text { (7) }\end{array}$} & \multicolumn{2}{|c|}{ Posgrado (3) } \\
\hline & $\begin{array}{l}\text { Inno- } \\
\text { vación }\end{array}$ & $\begin{array}{c}\text { Desem- } \\
\text { peño }\end{array}$ & $\begin{array}{l}\text { Inno- } \\
\text { vación }\end{array}$ & $\begin{array}{c}\text { Desem- } \\
\text { peño }\end{array}$ & $\begin{array}{l}\text { Inno- } \\
\text { vación }\end{array}$ & $\begin{array}{c}\text { Desem- } \\
\text { peño }\end{array}$ & $\begin{array}{l}\text { Inno- } \\
\text { vación }\end{array}$ & $\begin{array}{c}\text { Desem- } \\
\text { peño }\end{array}$ & $\begin{array}{l}\text { Inno- } \\
\text { vación }\end{array}$ & $\begin{array}{c}\text { Desem- } \\
\text { peño }\end{array}$ & $\begin{array}{l}\text { Inno- } \\
\text { vación }\end{array}$ & $\begin{array}{c}\text { Desem- } \\
\text { peño }\end{array}$ & $\begin{array}{l}\text { Inno- } \\
\text { vación }\end{array}$ & $\begin{array}{c}\text { Desem- } \\
\text { peño }\end{array}$ & $\begin{array}{l}\text { Inno- } \\
\text { vación }\end{array}$ & $\begin{array}{c}\text { Desem- } \\
\text { peño }\end{array}$ \\
\hline Menos de 2 años de experiencia & 1.00 & 4.00 & - & - & 4.17 & 4.75 & 1.00 & 1.00 & - & - & - & - & 4.75 & 4.00 & - & - \\
\hline De 2 a 3 años de experiencia & 1.00 & 4.00 & 1.00 & 2.00 & 5.17 & 5.63 & - & - & 4.00 & 3.50 & - & - & 2.00 & 2.67 & - & - \\
\hline De 4 a 5 años de experiencia & - & - & - & - & 4.00 & 4.00 & 3.58 & 4.75 & - & - & - & - & - & - & 5.00 & 6.33 \\
\hline Más de 20 años de experiencia & 4.33 & 5.50 & 4.17 & 5.50 & - & - & 4.67 & 6.00 & - & - & - & - & 3.58 & 5.00 & & - \\
\hline
\end{tabular}

Fuente: Elaboración propia.

Para el grupo de los administradores, los que obtuvieron menores puntajes en innovación, coinciden con los que obtuvieron puntajes bajos en desempeño: por una parte, los administradores con arquetipo defensor 
y carrera técnica que tienen entre dos y tres años de experiencia, y por otra parte los adminsitradores con arquetipo defensor que aunque han estudiado un posgrado, tienen menos de dos años de experiencia. Aunado a esto, se suman los bajos resultados de innovación obtenidos por los administradores con arquetipo defensor sin educación superior y con menos de tres años de experiencia.

En contraste, los administradores defensores con posgrado y con más de 20 años de experiencia, obtuvieron los mejores resultados en desempeño dentro del grupo de adminsitradores.

Finalmente, los administradores con arquetipo analista son, en su mayoría, personas que han cursado una licenciatura o posgrado. De este grupo, los que obtuvieron mejores resultados son los que tienen un posgrado y cuentan con 4 o 5 años de experiencia.

\section{Discusión de resultados}

La hipótesis planteada en esta investigación se pudo probar. Por un lado, se encontró una relación fuerte y significativa del nivel educativo del dueño y administrador con la innovación; por el otro, se encontró una relación fuerte y significativa entre la experiencia del dueño y administrador con el desempeño. Finalmente, para el grupo de los dueños, se encontró una relación fuerte y significativa entre el arquetipo estratégico y la innovación.

Los arquetipos estratégicos de Miles y Snow (1978) han sido un parámetro para caracterizar los distintos estilos de gestión entre las empresas. En este estudio se encontró que los dueños y administradores entrevistados, corresponden a los arquetipos defensores y analistas. Si bien es algo aplaudible que no tengan un arquetipo reactor con el que no podrían adaptarse rápidamente a las circunstancias del mercado, destaca el hecho de que no existan arquetipos prospectores, tal vez porque la experiencia les ha hecho tener cierta aversión a los riesgos y con ello, han logrado permanecer en el mercado, aunque sin muchas innovaciones. $\mathrm{Al}$ no encontrar diferencias significativas, no puede corroborarse que el grado de innovación de los analistas sea mayor que el de los defensores, como lo plantea McDaniel y Kolari (1987). Lo mismo sucede con los resultados de desempeño, pues no hay diferencias estadísticas entre los defensores y analistas. Este hallazgo corrobora lo descrito por Miles y Snow (1978), Conant, Mokwa y Varadarajan (1990) y Wright et al. (1991), pues los analistas y defensores demostraron ser igualmente exitosos en el desempeño de sus pequeñas organizaciones.

Cabe señalar que en las PYMES, el rol como dueño más que el del administrador, definen la orientación estratégica de estas organizaciones, razón por la cual la relación con la variable de arquetipos estratégicos fue significativa con la innovación, pero sólo en el grupo de los dueños. Por otra parte, en las PYMES los administradores están sujetos a las propuestas que autoricen los dueños, por lo que a pesar de que los administradores desarrollan arquetipos más proactivos, las PYMES no se beneficiarán en mayores niveles de innovación y desempeño si el dueño 
no aprueba las estrategias del administrador, por lo que seguramente y a pesar del adminsitrador- predominará el punto de vista del dueño (Arenius y Minniti, 2005; Grilo y Thurik, 2008).

A partir de los planteamientos anteriores, puede considerarse de manera detallada el caso de cada grupo analizado. En primer lugar están los dueños, grupo en el que demostraron ser más innovadores y creativos los que tienen un arquetipo analista, que tienen una licenciatura o posgrado y que tienen más de veinte años de experiencia. Este grupo debe inclinarse a la formación de la siguiente generación, a partir del involucramiento en los asuntos importantes de la PYME, en el que deberán acompañarse de un grupo de expertos que bien pueden contratar con otras PYMES del gremio. Los dueños que no cuentan con educación superior con poca o mucha experiencia, deben recurrir también a las enseñanzas de otros colegas del gremio, de sus propios clientes y de los consultores expertos. Al comprender su debilidad en la educación formal que carecen, pueden ser más receptivos y más abiertos a nuevas $\mathrm{e}$ innovadoras ideas.

Para el grupo de los dueños con arquetipo defensor, su principal fortaleza es la experiencia, pues los resultados demuestran que entre más experiencia adquieren, mejores resultados obtienen, aunque se observa una decadencia de buenos resultados cuando aucmulan más de 20 años en el cargo. Además, parece ser que cuando adquieren más estudios después de la licenciatura, no consiguen mejores resultados. A diferencia del estudio de Gray (2006) que encontró una relación significativa entre el nivel de estudios y la edad joven para una mayor innovación, en este estudio se hace evidente que los dueños con estudios superiores pero con una mayor experiencia, tienen una gama más amplia de contactos que utilizan para allegarse de información valiosa que analizan cuidadosamente para tomar la decisión de realizar innovaciones que les ayuda a impulsar niveles superiores de desempeño.

Por otra parte, los administradores con arquetipo estratégico analista, con posgrado y entre cuatro y cinco años de experiencia también obtuvieron buenos puntajes, porque son personas relativamente jóvenes para proponer y realizar innovaciones en productos y procesos productivos y tan seguros de sus acciones, que se ganan la confianza de los dueños y con ello, tienen mayor poder de decisión.

Los administradores que no cuentan con estudios superiores y que tampoco tienen un gran número de años de experiencia, difícilmente alcanzan niveles superiores de innovación y desempeño, pues al parecer, están gestionando de una manera empírica, lo que no les ayuda en tomar decisiones más informadas y concientes que pudieran orientarles en los cambios que requieren sus organizaciones.

Por otra parte, los administradores que tienen educación superior pero que son jóvenes, difícilmente pueden tener libertad de actuación, pues los dueños originales no confían totalmente en su capacidad, por su falta de edad, más que de experiencia. 


\section{Conclusiones}

Este estudio se examinan las características del dueño y administrador desde una perspectiva basada en la teoría de recursos y capacidades, analizando el efecto de los diferentes perfiles estratégicos, la experiencia y el nivel de estudios, tienen en la innovación y desempeño de las PYMES metalmecánicas de la industria automotriz del Valle de Toluca. A diferencia de la mayor parte de la literatura reconocida, esta investigación muestra estas relaciones en el contexto de las PYMES de un solo sector y de una sola región, buscando que la muestra sea más homogénea.

En este estudio se exploraron las diferencias entre los dueños y los administradores de las PYMES, obteniendo algunos hallazgos que permiten afirmar que el rol de quien dirige la empresa, es determinante para definir un arquetipo estratégico en particular y asumir mayor o menor riesgo para innovar y por ende, para buscar desempeños superiores. Por tanto, esta investigación proporciona nuevas pruebas sobre la influencia de las características de los dueños y administradores sobre la innovación y desempeño de las PYMES.

Los dueños que tienden a ser defensores sin experiencia, podrían trabajar en empresas grandes para construir una red de conocimientos con otras PYMES, clientes y proveedores, que les ayude a acelerar el proceso de aprendizaje. En contraparte, los dueños con mayor experiencia, deben abrirse a otros puntos de vista y pensar en la formación de la siguiente generación, involucrándolos en las operaciones y decisiones de la PYME, con el acompañamiento de expertos.

Los dueños que tienden a ser analistas y que tienen más de 20 años de experiencia, deben seguir cultivando redes de conocimiento con otros colegas y especialistas (Eisenhardt y Schoonhoven, 1996), con el fin de mantener altos niveles de innovación y desempeño.

Dado que las PYMES cuentan con pocos recursos para realizar investigación y por ende, realizan escasos esfuerzos para conocer y comprender su entorno, el aprendizaje es una manera de compensar la poca capacidad de adaptación que la mayoría tiene (Sirén y Kotamäki, 2015), por lo que los esfuerzos que los gobiernos federales deben realizar para apoyar a estas unidades económicas, debe enfocarse más al aprendizaje que al simple otorgamiento de créditos y apoyos financieros.

\section{Bibliografía}

Álvarez Medina, L. (2016). Formación de redes de conocimiento en México: cambios impulsados por la competencia en la industria automotriz mundial. Economía y Sociedad, (20). Recuperado a partir de http://economiaysociedad.umich.mx/ojs_ecosoc/index.php/ecosoc/ article/view/152

Apak, S., y Atay, E. (2014). Global Innovation and Knowledge Management Practice in Small and Medium Enterprises (SMEs) in Turkey and the Balkans. Procedia- Social and Behavioral Sciences, 150, 1260-1266. https://doi.org/10.1016/j.sbspro.2014.09.142 
Arenius, P. y Minniti, M. (2005). Perceptual variables and nascent entrepreneurship. Small Business Economics, 24(3), 233-247.

Aragón-Sánchez, A. y Sánchez-Marín, G. (2005). Strategic Orientation, Management Characteristics, and Performance: A Study of Spanish SMEs. Journal of Small Business Management. 43(3), 287-308

Avendano, R., Melguizo, A. y Miner, S. (2017). Chinese FDI in Latin America: New Trends with Global Implications. Washington, USA: Atlantic Council.

Bagnoli, C., y Vedovato, M. (2012). The impact of knowledge management and strategy configuration coherence on SME performance. Journal of Management \& Governance, 18(2), 615-647. https://doi.org/10.1007/ s10997-012-9211-z

Barbero, J.L., Casillas, J.C. y Feldman, H.D. (2011). Managerial capabilities and paths to growth as determinants of high-growth small and medium-sized enterprises. International Small Business Journal, 29 (6), 671-694

Barney, J. B. (1991). Firm Resources and Sustained Competitive Advantage. Journal of Management, 17, 99-120.

Baptista Nunes, M., Annansingh, F., Eaglestone, B., \& Wakefield, R. (2006). Knowledge management issues in knowledge-intensive SMEs. Journal of Documentation, 62(1), 101-119.

Bautista, S. J., y Peralta, C. M. R. (2017). La inclusión de las PyMEs en la Cadena de valor de la Industria Automotriz en México en el marco del Tratado Trans-Pacífico (TTP). Economía Informa, 403, 46-65.

Becker, W. y Peters, J. (2000). Technological opportunities, absorptive capacities, and innovation. Volkswirtschaftliche Diskussionsreihe, Institut für Volkswirtschaftslehre der Universität Augsburg. Recuperado a partir de https://www.econstor.eu/handle/10419/70043

Blackburn, R. A., Hart, M., y Wainwright, T. (2013). Small business performance: business, strategy and owner-manager characteristics. Journal of small business and enterprise development, 20(1), 8-27.

Camison, C. (1997). La Competitividad de la PYME Industrial Espan\#ola: Estrategia y Competencias Distintivas. Madrid: Civitas.

Cockburn, I. M. y Henderson, R. M. (1998). Absorptive capacity, coauthoring behavior, and the organization of research in drug discovery. The Journal of Industrial Economics, 46 (2), 157-182.

Cohen, W. M., \& Levinthal, D. A. (1990). Absorptive capacity: A new perspective on learning and innovation. Administrative science quarterly, $128-152$

Conant, J. S., M. P. Mokwa, and P. R. Varadarajan (1990). Strategic Types, Distinctive Marketing Competencies and Organizational Performance: A Multiple Measures Based Study. Strategic Management Journal 11, 365-383

Covarrubias Valdenebro, A. (2014). Explosión de la Industria Automotriz en México: De sus encadenamientos actuales a su potencial transformador. Fundación Friedrich Ebert en México. Recuperado a partir de http://www.fesmex.org/common/Documentos/Libros/ Paper_AP_Explosion_dela_Ind_Automortriz_AlexCovarrubias_Mar2014.pdf 
Delmar, F. y Wiklund, J. (2008). The effect of small business managers' growth motivation on firm growth: a longitudinal study. Entrepreneurship Theory and Practice, 32(3), 437-453.

DeSarbo, W., Di Benedetto, A., Song, M., y Sinha, I. J. (2005). Extending the miles and snow strategic framework: Strategic types, capabilities, environmental uncertainty, and firm performance. Recuperado a partir de https://papers.ssrn.com/sol3/papers.cfm?abstract_id $=893944$

Doty, D. H., Glick, W. H. y Huber, G.P. (1993). Fit, Equifinality, and Organizational Effectiveness: A Test of Two Configurational Theories, Academy of Management Journal. 36, 1196-1250.

Eisenhardt, K. M. Yschoonhoven, S. B., (1996). Resource-Based View of Strategic Alliance Formation: Strategic and Social Effects in Entrepreneurial Firms, Organization Science, 7, 136-150.

Estañol, A., Lara, R., Rodríguez, I., Sánchez, S., Sigler, E., Torres, E., \& Valle, A. (2016). Apocalyse not yet. Expansión, XLVIII(1202), 86-90.

Estrada, R., García Pérez, D. y Sánchez Trejo, V. G. (2009). Factores determinantes del éxito competitivo en la PYME: Estudio Empírico en México. Revista Venezolana de Gerencia, 14(46), 169-182.

González, Y. y Luna, C. (2016). Cómo superar la adicción al TLCAN. Expansión, XLVIII (1202), 84-90.

Grant, R. M. (1991). Contemporary Strategy Analysis: Concepts, Techniques, and Applications. Cambridge: Blackwell Publishers.

Gray, C. (2006). Absorptive capacity, knowledge management and innovation in entrepreneurial small firms. International Journal of Entrepreneurial Behavior \& Research, 12(6), 345-360.

Grilo, I. y Thurik, A.R. (2008). Determinants of entrepreneurial engagement levels in Europe and the US. Industrial and Corporate Change, 17(6), 1113-1145.

Hair, J. F., Prentice, E. y Cano, D. (1999). Análisis multivariante. Madrid: Prentice-Hall.

Hansen, B., y Hamilton, R. T. (2011). Factors distinguishing small firm growers and non-growers. International small business journal, 29(3), 278-294.

INEGI (2015). Principales resultados de la Encuesta Intercensal 2015. Recuperado de http://www.inegi.org.mx.

INEGI y AMIA. (2016). Estadisticas a propósito de... la industria automotriz. Aguascalientes, México: INEGI. Recuperado a partir de http:// www.amia.com.mx/index.html

Jaouen, A. y Lasch, F. (2013). A new typolgy of micro-firm owner-managers. International Small Business Journal, 0(0), 1-25

Marcketti, S., Niehm, L. y Fuloria, R. (2006). An exploratory study of lifestyle entrepreneurship and its relation-ship to life quality. Family and Consumer Sciences Research Journal, 34(3), 241-259.

McDaniel, S. W. y Kolari, J. W., (1987). Marketing Strategy Implications of the Miles and Snow Typology. Journal of Marketing, 51, 19-30.

Miles, R. E. y Snow, C. C. (1978). Organizational Strategy, Structure, and Process. New York: McGraw-Hill.

Navarrete, E. (2011). El fracaso de las micro, pequeñas y medianas empresas en Quintana Roo, México. Revista Internacional Administración \& Finanzas, 4,21 
Nieto, M. y Quevedo, P. (2005). Absorptive capacity, technological opportunity, knowledge spillovers, and innovative effort. Technovation, 25(10), 1141-1157.

Peteraf, M. A. (1993). The Cornerstones of Competitive Advantage: A Resource-Based View. Strategic Management Journal. 14, 179-191.

Pil, F. K., y Holweg, M., (2003). Exploring Scale. The Advantages of Thinking Small, MIT Sloan Management Review. 44(2),33-39.

Prahalad, C. K., y Hamel, G. (1990). The Core Competence of the Corporation. Harvard Business Review. 68(3), 79-91

Richbell, S., Brookes, M., Brewster, C. y Wood, G. (2011). Nonstandard working time: an international and comparative analysis. The International Journal of Human Resource Management, 22(04), 945-962.

Romanelli, E. y Tushman, M. L. (1994). Organizational transformation as punctuated equilibrium: An empirical test. Academy of Management Journal, 37(5), 1141-1166.

Rosenbusch, N., Brinckmann, J., \& Bausch, A. (2011). Is innovation always beneficial? A meta-analysis of the relationship between innovation and performance in SMEs. Journal of Business Venturing, 26(4), 441-457.

Schreiner, M., Kale, P., \& Corsten, D. (2009). What really is alliance management capability and how does it impact alliance outcomes and success?. Strategic Management Journal, 30(13), 1395-1419.

Sire\#n, C., y Kohtama\#ki, M., (2015). Stretching strategic learning to the limit: The interaction between strategic planning and learning, Journal of Business Research, http://dx.doi.org/10.1016/j.jbusres.2015.08.035

Stewart Jr, W. H., \& Roth, P. L. (2007). A meta\#analysis of achievement motivation differences between entrepreneurs and managers. Journal of Small Business Management, 45(4), 401-421

Subramaniam, M., \& Youndt, M. A. (2005). The influence of intellectual capital on the types of innovative capabilities. Academy of Management Journal, 48(3), 450-463.

van den Hooff, B., \& Huysman, M. (2009). Managing knowledge sharing: Emergent and engineering approaches. Information \& Management, 46(1), 1-8.

Vargas, I., (2012). El fracaso acecha a PYMES mexicanas. Revista Expansión. Recuperado de https://expansion.mx/emprendedores/2012/04/05/elfracaso-acecha-a-pymes-mexicanas

Veugelers, R. (1997). Internal R \& D expenditures and external technology sourcing. Research policy, 26(3), 303-315.

Vicencio Miranda, A. (2007). La industria automotriz en México: Antecedentes, situación actual y perspectivas. Contaduría y Administración, (221), 209-246.

Wernerfelt, B. (1984). A Resource-Based View of the Firm. Strategic Management Journal, 5, 171-180.

Wright, P., Kroll, M., Chan, P. y Hamel, K. (1991). Strategic Profiles and Performance: An Empirical Test of Select Key Propositions. Journal of the Academy of Marketing Science, 19, 245-254. 
Osvaldo Garcia Marin, et al. Características del dueño-administrador en la innovación y desempeño de pequeñas y medianas empresas metalmecán...

\section{Notas}

[1] Específicamente se recolectó información de PYMES localizadas en los municipios de Toluca, Metepec, Zinacantepec, San Mateo Atenco, Lerma y Tenango del Valle. Estos municipios constituyen gran parte del Valle de Toluca. 